\title{
Pharmacological and clinical overview of cloperastine in treatment of cough
}

This article was published in the following Dove Press journal:

Therapeutics and Clinical Risk Management

5 March 201I

Number of times this article has been viewed

\author{
Maria Antonietta Catania' \\ Salvatore Cuzzocrea ${ }^{1,2}$ \\ 'Department of Clinical and \\ Experimental Medicine and \\ Pharmacology, School of Medicine, \\ University of Messina; ${ }^{2}$ IRCCS \\ Centro Neurolesi "Bonino-Pulejo", \\ Messina, Italy
}

\begin{abstract}
Cough constitutes an impressive expression of the normal defense mechanisms of the respiratory system. Productive cough associated with catarrh is an important protective system for the lung because it favors the upward movement of secretions and foreign bodies to the larynx and mouth. Cough may also appear without bronchial secretions, as dry cough, which may be persistent when inflammatory disease is chronic or when, in the early stages of respiratory disease, bronchial secretions are not yet fluid. Sometimes bronchitis-induced cough does not significantly affect quality of life, whilst in other cases cough may become so intense as to impair daily activities severely, resulting in permanent disability. This type of cough is one of the most frequent reasons for seeking medical advice. The use of cough suppressants may be appropriate for reaching a precise diagnosis and when dry cough is persistent. Cloperastine has been investigated in various types of cough and, unlike codeine, has been shown to possess dual activity. It also acts as a mild bronchorelaxant and has antihistaminic activity, without acting on the central nervous system or the respiratory center. Here we review the preclinical and clinical evidence of the efficacy and tolerability of cloperastine.
\end{abstract}

Keywords: cough, cloperastine, inflammation, bronchitis

\section{Introduction}

Cough is a normal mechanism for the maintenance of a healthy respiratory system, ${ }^{1,2}$ and the nerves involved are located in two pathways, ie, an afferent limb and an efferent limb. ${ }^{3}$ The afferent pathways involve receptors located in the larynx, throughout the respiratory tree, on the pleura in the hearing channel, in the nose, and in the paranasal sinuses, pharynx, stomach, and diaphragm, and involve the trigeminal, glossopharyngeal, phrenic, and vagus nerves. ${ }^{4}$ Cough is characterized by an initial, short inspiratory phase, followed by closure of the glottis and rapid expiration with violent expulsion of air. ${ }^{5}$ In general, it is an important defense mechanism that expels infectious or excessive secretions from the tracheobronchial tree, and removes and dislocates exudates, cellular detritus, and foreign bodies ${ }^{5}$ (see Figure 1).

Management of a cough should be directed at the underlying cause. Thus, the first important step is to make a differential diagnosis in order to identify the cause of coughing and exclude serious pathology. Different studies have demonstrated the existence of different receptors for mechanical, chemical, and pharmacological stimuli, and these respond differently to antitussive agents. ${ }^{4}$ When productive and associated with catarrh, cough serves as an important protective system for the lungs, because it favors the upward movement of secretions and foreign bodies to the larynx and mouth. However, cough may also occur without fluid bronchial secretions, as dry 


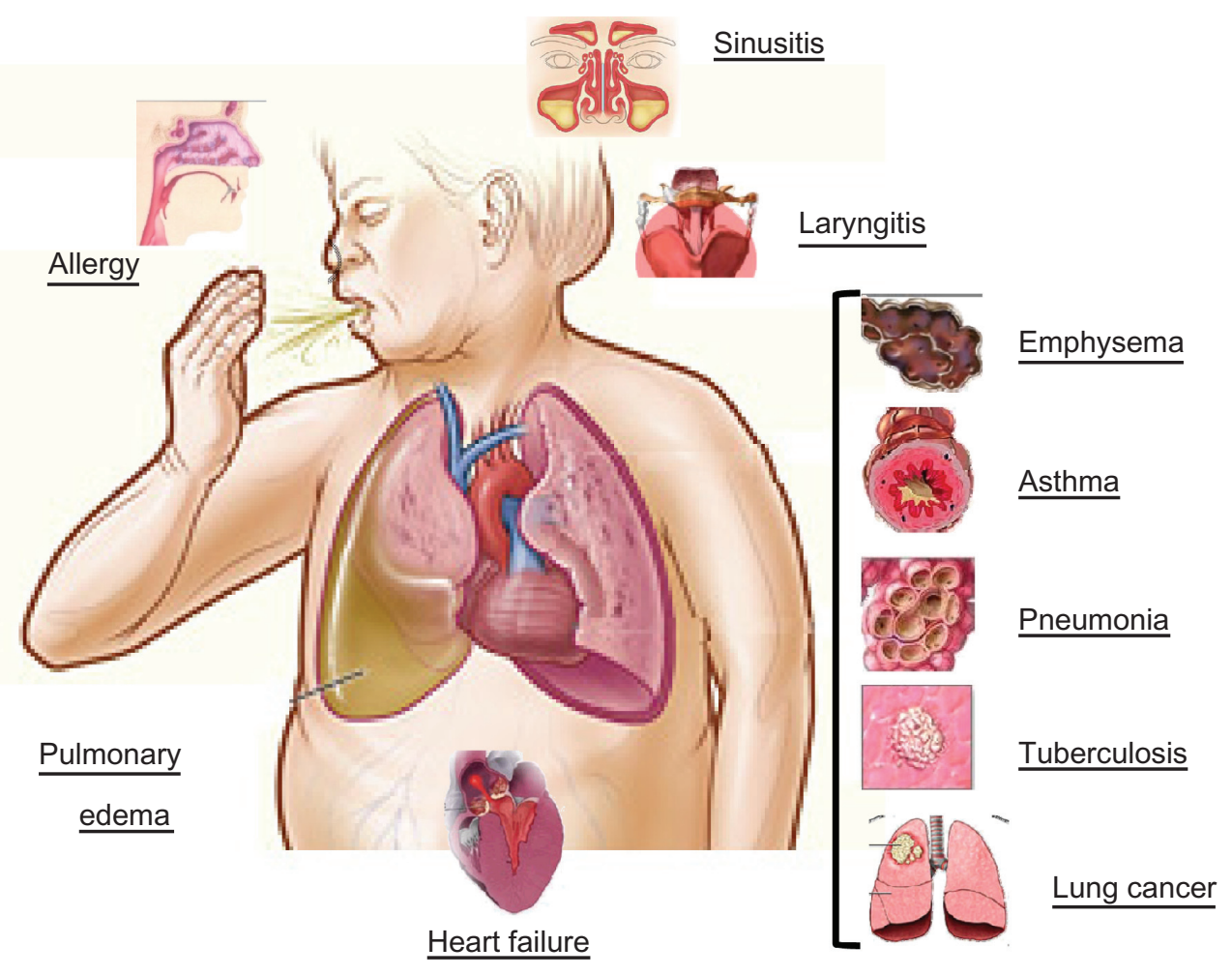

Figure I Some causes of cough

cough, which may be persistent when inflammatory disease is chronic or when, in the early stages of respiratory disease, bronchial secretions are not yet fluid. ${ }^{1}$ In most cases, cough occurs as part of a series of symptoms that pinpoints the site or organ involved in the morbid process. In the case of acute cough, an accurate case history and objective clinical examination provides a diagnosis without having to resort to laboratory tests. In the case of chronic cough, diagnostic tests are required to identify both the specific etiopathogenic causes and nonspecific stimulus factors. ${ }^{5}$

The most frequent causes of chronic cough are cigarette smoke, irritants from pollutants, bronchial and pulmonary carcinoma, asthma, chronic obstructive pulmonary disease (COPD), sinusitis, gastroesophageal reflux, iatrogenic and psychogenic cough, and, lastly, tuberculosis, which has returned as a health problem. ${ }^{4}$

Any disorder resulting in inflammation, constriction, infiltration, or compression of the airways can be associated with cough. Inflammation commonly results from various airway infections, ranging from viral or bacterial bronchitis to bronchiectasis. Another common cause of cough is asthma, that may presenting without wheezing or dyspnea, making the diagnosis more difficult.

Infiltrating airway wall cancer, granulomas (eg, sarcoidosis or tuberculosis), compression of the airways from extrinsic masses, interstitial lung disease, pneumonia, lung abscess, and congestive heart failure may be associated with cough. ${ }^{4,5}$ Sinusitis causes cough due to postnatal drip of secretions into the trachea, gastroesophageal reflux causes nocturnal reflex cough, and psychogenic cough is a common symptom of stress, and occurs particularly in people who often speak in public and in children. ${ }^{4}$

Nonproductive cough is also caused by the more widespread use of antihypertensive agents, such as the angiotensinconverting enzyme inhibitors in 5\%-20\% of patients taking these drugs, ${ }^{4}$ and is probably related to accumulation of bradykinin or substance $\mathrm{P}$, resulting from the pharmacological activity of this class of drugs. Usually the onset of angiotensinconverting enzyme inhibitor-related cough is within one week of starting the drug, but can be delayed. ${ }^{3}$

The clinical picture may help to confirm the diagnosis, eg, if other upper respiratory tract infection symptoms are present, such as rhinorrhea and sore throat. Coughing may be caused by infection (more likely of viral than bacterial etiology), croup, or tracheomalacia accompanied by a barking cough.

Pneumonia, and bronchiolitis may be accompanied by respiratory distress. Paroxystic cough suggests pertussis infection, and a temporal association with feeding or with positioning should indicate gastroesophageal reflux. ${ }^{2}$ 
Duration of the cough may be helpful:

- Acute cough (for less than three weeks before diagnosis) is most often due to upper respiratory infection, but more serious diseases, such as pneumonia, pulmonary embolism, or congestive heart failure, cannot be ruled out

- Subacute cough (for 3-8 weeks) is more commonly postinfectious, due to persistent airway inflammation and/or postnasal drip

- Chronic cough (more than 8 weeks) may suggest COPD or bronchogenic carcinoma in smokers, while in nonsmokers (not taking angiotensin-converting enzyme inhibitors and with a normal chest radiograph) the most common causes of chronic cough are postnasal drip, asthma, and gastroesophageal reflux. ${ }^{3}$

Symptomatic treatment with antitussives is usually employed if the cough is nonproductive, barking, and significantly interferes with the well-being of the pediatric or adult patient (causing, eg, nausea, vomiting, insomnia, and/or headache). Antitussives can be divided into two main categories depending upon whether the mechanism of action is central or peripheral. Central antitussives include narcotics and non-narcotics, and act more or less selectively on the cough center located in the medulla oblongata. Non-narcotic therapy is preferable in children, because narcotic antitussives (eg, levorphanol, codeine) may cause respiratory center depression, nausea, vomiting, constipation, addiction, and physical dependency. ${ }^{5}$

The therapeutic strategy should treat the cough with suitable pharmacological therapy, taking into account the nature and etiology of the cough. In addition, the treatment should have a sustained action throughout the day, and should preserve sleep quality at night time. ${ }^{4}$

Mucolytics are often prescribed as adjuvant therapy to eliminate catarrh in chronic and acute bronchitis, bronchial asthma, and bronchiectasis. ${ }^{4}$ Some of the most common ones used are cysteine derivatives, eg, $N$-acetylcysteine, which can break the sulfide links between glycoproteins and loosen mucus. ${ }^{6,7}$ Antitussives are mainly used to treat chronic persistent dry cough when the etiology is a chronic pathology. ${ }^{8-10}$ Among the antitussive agents, cloperastine, which possesses antitussive and antiedemic activity, also relaxes the bronchial musculature. Unlike codeine, cloperastine is not a narcotic, acts directly on the cough center, does not possess local anesthetic activity, and does not depress the respiratory center. ${ }^{8}$

Some of the centrally acting opioid derivatives used today include dextromethorphan, noscapine, codeine, and dihydrocodeine, which may lead to dependency and, in rare cases, halt breathing, especially in the elderly. ${ }^{4}$ Even though they are often available as commercial syrups, expectorants, and antitussives, these products should not be used together because they counteract each other. ${ }^{4}$

The US Food and Drug Administration (FDA) has advised against the use of over-the-counter cough and cold medicines in children younger than two years of age, and recommended caution when using such medicines in children aged 2-11 years because of the risk of life-threatening adverse effects (FDA Public Health Advisory 2008). It is preferable not to use these agents in children of any age, particularly in the presence of neurological disorders, seizures, hypotonia, heart disease, and in view of the risk of respiratory depression (see FDA warning).

Therefore, it is imperative to check whether it is appropriate to prescribe cough and cold medicines for children. For pediatric patients is no reliable evidence to recommend cough and cold medicines in children younger than two years of age, although most clinical studies have been performed in older children. ${ }^{2}$

In addition, a Cochrane review ${ }^{9}$ including two trials with antitussives (dextromethorphan and codeine), two with antihistamines (clemastine and chlorpheniramine, diphenhydramine), two with antihistamine-decongestants (brompheniramine-phenylbropanolamine and brompheniramine-phenylephrine-propanolamine), and one with an antitussive-bronchodilator combination (dextromethorphan-salbutamol) found that these treatments were no more effective than placebo for acute cough in children. Another Cochrane review found insufficient evidence to establish whether over-the-counter medicines were helpful for cough when associated with antibiotic use for acute pneumonia in children and adults. ${ }^{10}$ Moreover, although most of the trials included in the Cochrane reviews did not report adverse events, it is well known that cough and cold products in children are a major cause of unintentional drug overdose, ${ }^{11}$ and are associated with mortality. Indeed, during 2004-2005, 1519 children younger than two years of age were treated in US emergency departments for adverse events associated with cough and cold medications. Because of the risk of toxicity, lack of dosing recommendations, and limited evidence for the effectiveness of these drugs in children younger than two years, clinicians should exercise caution when prescribing them, and warn parents and caregivers about these concerns. ${ }^{12}$

\section{Cloperastine}

Cloperastine (1-[2-(p-chloro-alpha-phenylbenzyloxy)ethyl] piperidine) is a drug with a central antitussive effect, and is also endowed with an antihistaminic (sharing an ethylamine 
moiety with $H_{1}$ receptor antagonists) and papaverine-like activity similar to codeine but without its narcotic effects. Pharmacological studies have shown that the molecule acts on the cough center without depressing the respiratory center, and that it has no negative cardiocirculatory effects. ${ }^{13}$ The initial response at the therapeutic dose range (10-20 mg three times daily for adults) is 20-30 minutes after oral administration. Moreover, the duration of action of a single dose of cloperastine is 3-4 hours. Further pharmacokinetic information is provided in the Table 1 .

\section{Preclinical studies}

Cloperastine was first studied by Takagi et al at the University of Tokyo. ${ }^{14}$ This research group investigated the pharmacological activity of several derivatives of diphenhydramine, an antihistamine substance, and discovered that cloperastine had the greatest efficacy against cough caused by tracheal mechanical stimulus in the guinea pig. This effect was 1.9 times greater than codeine, without any narcotic effects.

In a later study conducted in dogs and cats, the same researchers showed that the activity of cloperastine specifically involved the cough center, without interfering with the respiratory center. Cloperastine, which also possesses bronchorelaxant and antihistaminic activity, helped to relax the bronchial musculature which had become tight due to stimulation by acetylcholine and histamine. ${ }^{14}$ In particular, it was shown that cloperastine was able to induce a significant and dose-dependent reduction in the number of coughing fits induced in guinea pigs by $\mathrm{NH}_{3}$ or citric acid vapor, and its antitussive activity appeared similar to that of codeine in this experimental model. The antihistaminic effect of cloperastine is demonstrable in guinea pigs by a histamine aerosol-induced bronchospasm test. ${ }^{14}$ The drug is able to antagonize tracheal chain contractions induced by various agonists in guinea pigs, thus having a muscle relaxant effect, particularly remarkable in histamine-induced contractions, but appears to be free of any influence on spontaneous motility. ${ }^{14}$ Cloperastine also shows good toxicological characteristics, without addiction potential, thus having promise for therapeutic use. ${ }^{14}$ In rats, the drug is metabolized in the liver and kidneys, where most of it is eliminated within 24 hours of administration. The drug reaches maximal concentration in the liver within two hours of ingestion, and is mainly eliminated by glycuronoconjugation. ${ }^{14}$ Cloperastine hydrochloride shows relatively low acute toxicity when administered by the intraperitoneal route in rats and mice, and shows minor toxicity by the oral route when administered as cloperastine fendizoate, the $\mathrm{LD}_{50}$ in rats and mice for the two administration routes exceeds 1000 and $2000 \mathrm{mg} / \mathrm{kg}$, respectively. ${ }^{14}$ The acute toxicity picture seen is mainly localized at level of the central nervous system, including depression and sedation. Chronic toxicity tests performed in rats treated for three months with cloperastine hydrochloride $15 \mathrm{mg} / \mathrm{kg}$ and $45 \mathrm{mg} / \mathrm{kg}$ and cloperastine fendizoate $100 \mathrm{mg} / \mathrm{kg}$ and $300 \mathrm{mg} / \mathrm{kg}$, and in dogs treated for three months with cloperastine hydrochloride $20 \mathrm{mg} / \mathrm{kg}$ and cloperastine fendizoate $100 \mathrm{mg} / \mathrm{kg}$, revealed no particular symptomatology or variations in hematochemical, hematological, and urinary parameters with respect to control animals and baseline values (unpublished data). Post mortem as well as macroscopic and microscopic examination also showed no evidence of pathological changes affecting the main organs and parenchyma. ${ }^{14}$

The possible activity of cloperastine in terms of both fetal toxicity and teratogenesis in rats and rabbits was also considered. Such tests did not demonstrate significantly different effects from those observed in control animals. Of interest are the pharmacokinetic characteristics of cloperastine, which reaches plasma levels within one hour of administration. Eight hours after intake, the drug is still detectable in plasma and for about six hours in tissues and parenchyma. It is completely metabolized in a short time and is mainly excreted in the urine as metabolites. It is in this context that the investigations performed by Takagi et al took place, aiming to evaluate the pharmacodynamic activity of the molecule, and in particular its antitussive effect. ${ }^{14}$ Cloperastine proved to reduce the number of

Table I Pharmacokinetics of cloperastine*

\begin{tabular}{|c|c|}
\hline \multirow[t]{2}{*}{ Onset and duration } & Initial response oral: $20-30$ minutes (Prod Info Nitossi ${ }^{\circledast}, 1997$ ) \\
\hline & Duration single oral dose: $3-4$ hours (Prod Info Nitossil ${ }^{\circledR}, 1997$ ) \\
\hline Drug concentration levels & Time to peak concentration oral tablet: $60-90$ minutes $^{15}$ \\
\hline Adsorption & Rapidly absorbed from oral administration (Prod Info Nitossi ${ }^{\circledR}, 1997$; Prod Info Quik ${ }^{\circledR}$ 1997) \\
\hline Metabolism & Extensive hepatic metabolism (Prod Info Seki $\left.{ }^{\circledR}, 1986\right)$ \\
\hline \multirow[t]{2}{*}{ Excretion } & Renal excretion; metabolites are eliminated by renal excretion ${ }^{15}$ \\
\hline & 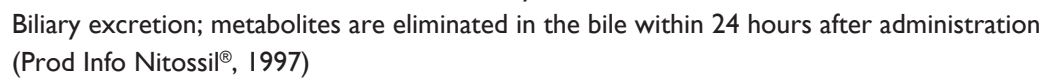 \\
\hline
\end{tabular}

Note: *Micromedex, last access October 25, 2010. 
coughing fits induced in guinea pigs significantly, even at a dose of $1 \mathrm{mg} / \mathrm{kg}$, and this activity was shown to be superimposable on that of codeine. With regard to potential antihistaminic action, the drug was shown to have high antihistaminic activity in the histamine aerosol-induced bronchospasm test. ${ }^{14}$ This activity, which was greater at the dose of $5 \mathrm{mg} / \mathrm{kg}$, was present also at the dose of $1 \mathrm{mg} / \mathrm{kg}$, thus demonstrating remarkable efficacy as an antihistaminic agent. ${ }^{14}$ In addition, both the hydrochloride and fendizoate salts of cloperastine were able to induce a significant and dose-dependent increase in sleep duration, but failed to increase significantly the time taken to disappearance of the righting reflex. ${ }^{14}$ Finally, the addiction potential of cloperastine was studied in animals addicted to morphine. These tests did not show a particular involvement of spontaneous motility in rats during four hours of recording, whilst codeine induced a marked increase in motility during the first hour of treatment. This is particularly important chronically, because it demonstrated a cross-dependence between codeine and morphine, whilst cloperastine induced no morphine-like dependence. ${ }^{14}$

\section{Cloperastine in treatment of chronic cough}

Many patients suffer chronically from this disturbing and debilitating symptom caused by the excessive production of mucus or an increased central response reflex. However, cough must be treated if its intensity becomes excessive and debilitating. Nonetheless, symptomatic treatment is difficult, and although drug therapy should target the etiology of the illness (eg, treatment with antibiotic and/or antiasthmatic therapy), in most cases this will not alleviate cough if there is no underlying cause.

Therefore, definitive treatment for cough depends primarily on its specific cause and the adoption of a specific therapy. Even if the etiologic therapy induces symptomatic remission, a nonspecific antitussive therapy may be required, especially in a patient suffering from a chronic disease.

It is true that the cough mechanism in chronic bronchitis partially compensates for the minor efficacy of the mucociliary depuration mechanism, which appears particularly impaired in these patients, due to the reduction in numbers of cilia. For this reason, the use of cough sedatives is not recommended for patients with chronic bronchitis in the exacerbation phase and with copious expectoration. ${ }^{8-10}$

Various studies have demonstrated clearly that cloperastine, which is free of narcotic activity and does not depress the respiratory center, has significant therapeutic activity against cough due to various pathologies, causing no respiratory depression and not interfering with protective mucociliary mechanisms. ${ }^{15}$ In a clinical study, Camisasca et a ${ }^{15}$ evaluated cloperastine in 23 patients with scarcely productive but persistent cough. Cloperastine $20 \mathrm{mg}$ as a single evening dose was chosen to enable better night-time rest by eliminating coughing fits and avoiding, in the meantime, depression of the tracheobronchial depuration mechanism during the day time. The majority of the treated patients showed a significant subjective improvement, documented by a clear reduction in previously troublesome night-time cough. No concomitant changes in partial oxygen or carbon dioxide pressures were reported, confirming that cloperastine does not have a negative effect on respiratory function.

Furthermore, Olivieri et al evaluated the efficacy of cloperastine in a double-blind study of 30 patients with chronic bronchopneumonia, tuberculosis, or bronchopulmonary malignancy, selected based on their cough frequency and intensity. ${ }^{13}$ The patients were randomized to receive cloperastine or placebo at the dose of 20 drops (equivalent to $35.4 \mathrm{mg}$ of the active substance) three times per day for nine days. The investigators clearly demonstrated the ability of cloperastine to reduce and control cough symptoms for the entire duration of the study. Improvement in cough, in turn, had a positive effect on night-time rest. Cloperastine does not appear to affect the volume, appearance, and viscoelastic properties of sputum, which underwent similar, positive changes in both groups of patients. This effect is attributable to the activity of baseline therapy during the hospital stay. The progression of respiratory rate and spirometric index values was also consistent, as were blood pressure and heart rate values, demonstrating that the antitussive effect of the drug is not accompanied by changes in cardiorespiratory function. ${ }^{13}$

Furthermore, in another clinical study of 21 patients with a range of bronchopulmonary illnesses and presenting with cough that required antitussive therapy, Fabris evaluated the efficacy of cloperastine administered for an average of 9 (range 3-16) days. ${ }^{16}$ However, based on the fact that the patient cases in the study varied in severity and diagnosis, the activity of the drug was rated by considering the individual patients on the one hand and the overall results on the other. Fabris demonstrated that, in most cases, $40 \mathrm{mg}$, or one $10 \mathrm{mg}$ dose of cloperastine twice per day plus two pills at bedtime, was sufficient to control cough, including throughout the night. ${ }^{16}$ Considering the overall score as described by Fabris, ${ }^{16}$ there was a significant reduction in cough as early as one day after the start of treatment, with a further subsequent reduction. This reduction was attributed not only to the efficacy of the drug, but also due to amelioration of the illness as it ran its course. Almost none of the patients had cough by day 11 of the study. Despite complete resolution of 
cough, treatment was continued for a few days in some patients because they still required some pharmacological cover. Reporting the results of this study in percentages, $66.7 \%$ of the subjects showed a maximum effect, $28.6 \%$ had a fair-satisfactory effect, and $9.5 \%$ had no effect. Moreover, cloperastine was shown to be particularly effective even in the most severe cases, such as lung cancer. ${ }^{16}$ Tolerability was excellent in all cases; only one subject could not be evaluated because treatment was stopped after three days due to lack of efficacy of the drug. There were no side effects, such as dry mouth, constipation, or intestinal problems. There were no statistically significant changes in blood pressure, heart rate, or any of the other biohumoral parameters studied. This research confirms the therapeutic efficacy and tolerability of cloperastine. It should also be pointed out that none of the patients experienced respiratory depression, and some subjects experienced antispastic bronchial activity while taking the drug. Another interesting aspect of the activity of cloperastine observed in this study was that it does not negatively affect the amount of mucus produced or its fluidity. ${ }^{16}$

In another double-blind investigation, cloperastine was shown to be effective as an antitussive in four tracheostomized patients, with no reported side effects. ${ }^{17}$ In particular, the authors of this study also pointed out that the two formulations of cloperastine used (ie, sugar-coated tablets and syrup) did not differ from each other in activity.

The beneficial effect of cloperastine has also been demonstrated in a multicenter, randomized, placebo-controlled study in 100 patients suffering from chronic or persistent dry or productive cough, resulting from acute and chronic respiratory tract disease. ${ }^{1}$ In this study, the authors reported significant subjective improvement, demonstrated by reduction of cough frequency and severity and a decrease in night-time cough, together with improvement in night rest, in patients treated with cloperastine three times daily and with a double dose in the evening. ${ }^{1}$

Importantly, a recent study has demonstrated the existence of different receptors for mechanical, chemical, and pharmacological stimuli, which respond differently to antitussive agents. ${ }^{4}$ Codeine remains the standard antitussive therapy but may lead to dependency and, in rare cases, halt breathing, especially in the elderly. In a controlled clinical study, Margarino et al evaluated the activity of cloperastine versus codeine in a "intent to treat" population of patients who were being monitored for a variety of reasons, and all had persistent chronic cough. ${ }^{4}$ This study randomized 156 patients to cloperastine syrup or dihydrocodeine, and it was reported that cloperastine had more significant activity. Moreover, the authors recommended that, because the com- parator was codeine and that the effects were measured in patients with an extensive case history of illness, cloperastine should be considered among the drugs of first choice for cough. Cloperastine meets the requirements of rapid action, maximum manageability, tolerability, and improvement in the quality of sleep, the latter being a very important feature in pediatric and debilitated adult patients. The authors suggested that cloperastine could be used safely for four days at a time. ${ }^{18}$ Furthermore, in another controlled clinical study ${ }^{19}$ in 38 outpatients with cough of various causes treated with codeine or cloperastine pills/chewable tablets, the efficacy of cloperastine in the symptomatic treatment of cough was also confirmed.

Importantly, cloperastine is as active as codeine, which has been the drug of choice for treating cough for years due to its overall sedative action on cough. In addition, cloperastine is highly effective for improving night-time rest disturbed by cough. These results confirm the value of the double dose at night time. Unlike codeine, cloperastine has an additional mild bronchorelaxant effect, which is useful in patients presenting with spastic airways. Both cloperastine preparations were shown to be equally active, improving compliance with treatment because the patient can choose the most suitable formulation. ${ }^{19}$

Finally, in a controlled, double-blind, clinical study, Ghiringhelli investigated the efficacy of cloperastine in patients with exacerbation of COPD. ${ }^{20}$ In the exacerbation phase of COPD, cough frequently appears as not purposive, namely nonproductive and wearying. This symptom therefore requires specific pharmacological intervention, enabling rapid control, while waiting for antibiotic therapy to treat the underlying cause. In such circumstances, evaluation of the efficacy of an antitussive preparation should take into account the response of the underlying disease to antibiotic therapy. To this end, the authors compared the effects of cloperastine with those of butamirate under analogous experimental conditions and at full therapeutic dosage and proven efficacy. This enabled the evaluation of cloperastine alone and also an evaluation of any possible difference between the effects of cloperastine and butamirate. The study of the "acute" effect, namely the response to the first dose of the drug, a parameter that can be considered to be independent of the course of the underlying disease, showed that cloperastine could reduce cough frequency and intensity. The superiority of cloperastine compared with butamirate was clinically evident and statistically demonstrable on the first and second days of treatment, taking into consideration the three symptom-related parameters examined (ie, frequency 
and intensity of cough during the day, rest disturbed by cough during the night). Finally, it is interesting to observe that, from the third day onwards, there was no statistically significant difference in cough control in the two groups, which may be the time frame in which antibiotic therapy takes effect and helps to bring cough under control. Thus, cloperastine was very effective as an antitussive agent in patients with exacerbation of COPD, allowing satisfactory symptomatic control from the first day of treatment in $65 \%$, from the second day in $20 \%$, and from the third day in $10 \%$ of cases. A useful therapeutic effect was therefore achieved in $95 \%$ of cases. Cloperastine administered three times daily was shown to be able to control cough effectively, not only during the day time but also during the night time. In addition, cloperastine has excellent tolerability, with no undesirable effects reported either at a clinical or biohumoral level. ${ }^{20}$ In conclusion, the results of all these studies confirm the favorable therapeutic index of cloperastine, and position it among the drugs of first choice for conditions in which a cough sedative is required.

\section{Cloperastine for children with chronic cough}

Respiratory tract infection is one of the most common causes of illness in children, and cough and fever are the most frequent symptoms that worry parents. Symptomatic therapy is appropriate in the case of a pointless, nonproductive, persistent cough caused by bronchial irritation, which often greatly disturbs the well being of young patients, and can be associated with nausea, vomiting, insomnia, and headache. ${ }^{5}$ Non-narcotic therapy is preferable in children because narcotic antitussives (codeine, dihydrocodeine) may cause respiratory depression, nausea, vomiting, constipation, addiction, and physical dependency. The clinical studies described earlier have clearly demonstrated that cloperastine acts directly on the cough center without depressing the respiratory center and without affecting the cardiocirculatory system. ${ }^{5}$

It is widely agreed that it is necessary, if not imperative, to conduct controlled, double-blind clinical studies to document the efficacy of symptomatic drugs such as antitussives specifically in children. Scotti and Borzani ${ }^{5}$ undertook a controlled, double-blind clinical efficacy study in children with cough secondary to acute bronchitis, and also attempted to define the optimal dose of cloperastine in accordance with the age and/or weight of the patients treated. The latter aspect is particularly important, because pediatric therapy must be administered in accordance with patient weight. This study had several important findings. Cloperastine was effective for settling irritative cough secondary to acute bronchitis. Cough intensity was reduced, expectoration was facilitated, and there was a significant improvement in auscultatory findings, confirming the efficacy of cloperastine. Lastly, a particularly valuable result was the posology used, which consisted of three administrations per day with a double dose in the evening, increasing the effect of the drug during the night to render sleep more restful and improving the overall well-being of the child. ${ }^{5}$

Similarly, in a single-blind study of 30 children aged 5-12 years with respiratory tract inflammation, Cicchetti ${ }^{21}$ confirmed that cloperastine is a highly effective treatment for children with persistent cough interfering with night-time rest. The tolerability of cloperastine was consistently good. ${ }^{20}$

Furthermore, in a randomized, double-blind, placebocontrolled study, Svitaylo evaluated the clinical efficacy and tolerability of cloperastine in children with nonproductive cough caused by respiratory tract infection. ${ }^{22}$ The study enrolled 200 patients, and included a placebo group to define the activity of the drug better. The dosage regimen and duration were the same as those normally used for cloperastine in clinical practice. The results of this study confirmed the antitussive activity of cloperastine fendizoate in children and adolescents with upper and lower respiratory tract infection in whom a nonproductive, persistent cough was one of the key symptoms that caused medical help to be sought. The therapeutic activity of cloperastine, which had already been evaluated in other controlled clinical studies, was investigated for both primary endpoints (cough intensity and frequency) and secondary endpoints (interference in duration and quality of sleep for the patient and parents). At the final visit, cough was absent in $80.4 \%$ of patients treated with cloperastine versus $10.4 \%$ of patients treated with placebo. Reduction of cough, which was homogenous in both arms of the study at the baseline visit, occurred rapidly in the cloperastine group, and the frequency of night-time cough, which made parents particularly anxious, decreased rapidly within the first three days of treatment. However, at various time points, mean scores were similar to those found during the first night of evaluation in the placebo arm, reinforcing that the improvement in cough seen in the cloperastine arm was due to the pharmacological activity of the drug and not attributable to spontaneous cough reduction.

Improvement or disappearance of cough is obviously reflected in quality of sleep for the child and parents, and examination of these endpoints showed a significant reduction in mean scores obtained in the cloperastine arm versus the placebo arm. The significant improvements 
obtained in all the endpoints evaluated were confirmed by the overall rating of the principal investigator who, blinded to the study, rated cloperastine treatment as excellent in $90.2 \%$ and good in $9.8 \%$ of subjects. The difference in the positive rating obtained in the placebo arm was very significant, both statistically and clinically.

The frequency of respiratory illnesses from colds, which is particularly high in children during winter, is often accompanied by a dry, barking cough leading to the widespread use of antitussives. Parents contribute to this by independently administering antitussives in order to alleviate the frequency and intensity of the cough, which has a negative effect on their quality of sleep, too, and thus on the performance of their daily activities. ${ }^{22}$ On the other hand, the literature contains ambiguous information about the activity and tolerability of these drugs. Codeine and dextromethorphan are widely used as antitussives and are not recommended by the American Pediatric Association due to a lack of conclusive proof of their benefit and their potential toxicity. ${ }^{23,24}$ Dextromethorphan is a centrally acting opioid which is widely used as an antitussive. It has been shown to be effective in children with upper respiratory tract infection, but more recent studies have found it to be no better than placebo for controlling acute cough. Even studies in adults with upper respiratory tract infection have not provided consistent results, and there have also been conflicting results for the antitussive activity of diphenhydramine, an antihistamine.

Of particular interest is a recent US study conducted in children with cough caused by upper respiratory tract infection, which evaluated the antitussive activity of dextromethorphan, diphenhydramine, and placebo on the frequency and severity of cough in pediatric patients and on the interference of this symptom with their sleep and the sleep of their parents. Despite improvement being found in all three groups for the endpoints evaluated, neither diphenhydramine nor dextromethorphan produced a significantly superior benefit over placebo. ${ }^{23}$

The literature reports various adverse events associated with the use of antitussives. Dystonia, ${ }^{25}$ anaphylactic reaction, ${ }^{26}$ and bullous mastocytosis ${ }^{27}$ have been seen with dextromethorphan, and this drug has also been abused $^{28,29}$ by young adolescents. Therapeutic doses of diphenhydramine have been associated with sleepiness, and also nervousness, restlessness, insomnia, acute dystonia, impaired driving ability, and increased risk of serious injury with standard doses. Adverse events such as nausea, vomiting, vertigo, sedation, palpitations, pruritus, and constipation have been seen with antitussive doses of codeine and dihydrocodeine, ${ }^{18}$ and potentiation phenomena with other centrally acting sedatives, such as anxiolytics and tricyclic antidepressants, have also been seen. The results of a placebo-controlled study conducted in a large number of patients demonstrate that cloperastine is among the drugs of first choice for the treatment of cough in children and adolescents with upper respiratory tract infection due to its efficacy, rapid action, and tolerability. Of particular interest is the effect of the drug on night-time sleep, with improvement in quality and duration of sleep and night-time rest, and also that of parents. In this regard, Seidita et al have evaluated the efficacy and tolerability of cloperastine syrup in a controlled clinical pediatric study versus control therapy based on syrup containing codeine and phenyltoloxamine for a total of seven days. ${ }^{30}$ The use of a well established and effective comparator drug in this study led to a more accurate and thorough evaluation of the antitussive activity of cloperastine. The results showed that both drugs had a positive effect on cough, but a comparison of the treatments demonstrated that cloperastine reduced the cough symptom more rapidly, which obviously led to a faster improvement in night-time rest, with better well-being in the children. It is important to control the cough symptom rapidly in these inflammatory respiratory illnesses if it is particularly severe while waiting for the therapy to cure the underlying cause. Respiratory rate and spirometric index measurements in all patients returned into the normal range, accompanied by an improvement in the bronchial airways due to the basic therapy, which confirmed that the two drugs do not depress the respiratory center at the therapeutic dosages used. In fact, probably due to its spasmolytic action in the bronchial airways, cloperastine seems to produce an earlier and more consistent improvement in respiratory function which is very slightly ascertainable, if at all, in the spirometric tests performed only at the end of treatment.

In a clinical study of 20 patients aged 2-14 years, Barbato et al also demonstrated that cloperastine was immediately effective in reducing cough, and acted particularly rapidly in night time cough, and significantly improved night-time rest of the children. ${ }^{31}$ The drug was well tolerated in this study due to lack of changes in respiratory and cardiocirculatory parameters, which was also confirmed by the laboratory data. The therapeutic activity of cloperastine is thus similar to codeine but without serious side effects, ie, nervousness, agitation, confusion, and convulsions. ${ }^{31}$ 


\section{Efficacy and safety summary}

Clinical studies have shown that cloperastine is well tolerated and effective in the treatment of cough due to various etiologies, decreasing the frequency and intensity of cough of this bothersome symptom, albeit in small patient populations. ${ }^{15-19,32}$ Moreover, cloperastine has shown potential advantages over opioid antitussives, which frequently cause sedation and respiratory depression, having comparable activity, but without the typical undesirable side effects. ${ }^{5}$

The efficacy of cloperastine was compared with the analog, levocloperastine, in open clinical trials, and demonstrated a comparable efficacy and tolerability profile. ${ }^{33}$

However, it is important to monitor signs and symptoms, ie, relief of cough, dyspnea, quantity and quality of sputum, ${ }^{17-19,34}$ respiratory rate, forced expiratory volume in one second, and residual volume. ${ }^{13}$ Moreover, in children it is appropriate to monitor body weight, cardiac and respiratory rate, blood pressure, sputum quality, dyspnea, and difficulty in expectoration. ${ }^{5}$

Finally, there has been one case report of a patient with acute dystonia associated with ingestion of cloperastine syrup, who also suffered from schizophrenia but had been neuroleptic-free for six months, suggesting a possible alteration of the balance between dopamine and acetylcholine in the striatum. ${ }^{35}$

\section{Conclusion}

Based on the clinical evidence, cloperastine is an effective drug for cough, after excluding or treating underlying causes. Its activity appears to be rapid and the drug contributes to improvement in the clinical picture and well-being of patients, as well as improvement in night-time sleep. Finally, its good tolerability and availability in syrup, drop, and tablet formulations (the last being only for adult administration), confirm that cloperastine can be used in a wide selection of the population (children, adolescents, and adults), according to the indications on the labeling and appropriate case-by-case evaluation. The efficacy and safety of cloperastine in the approved indication and recommended dose range have now been demonstrated during more than 40 years of clinical use.

\section{Disclosure}

The authors report no conflicts of interest in this work.

\section{References}

1. Margarino G. Efficacy and tolerability of cloperastine fendizoate in the treatment of different etiology chronic cough. Internista. 2002;10: 153-159. [Italian].

2. Sung V, Cranswick N. Cough and cold remedies for children. Australian Prescriber. 2009;32:122-124.
3. Fauci AS, Braunwald E, Kasper DL, et al. Harrison's Principles of Internal Medicine. 17e.

4. Margarino G, Scala M, Nocentini L. Cloperastine in the treatment of persistent chronic cough. A controlled clinical study. JAMA. 1998;10: 3-7. [Italian version].

5. Scotti L, Borzani M. Cloperastine fendizoate in the treatment of coughproducing diseases in pediatrics. Minerva Pediatr. 1988;40:283-286.

6. Gallon AM. Evaluation of nebulized acetylcysteine and normal saline in the treatment of sputum retention following thoracotomy. Thorax. 1996;51:429-432.

7. Moldéns P, Cotgreave IA, Berggren M. Lung protection by a thiolcontaining antioxidant: N-acetylcysteine. Respiration. 1986;50 Suppl 1: $31-42$.

8. Olivieri D, Del Donno M, Ferraro E. Effect of cloperastine on mucociliary clearance. Arch Monaldi. 1983;38:219-223. [Italian].

9. Smith SM, Schroeder K, Fahey T. Over-the-counter medications for acute cough in children and adults in ambulatory settings. Cochrane Database Syst Rev. 2008;23:CD001831.

10. Chang CC, Cheng AC, Chang AB. Over-the-counter (OTC) medications to reduce cough as an adjunct to antibiotics for acute pneumonia in children and adults. Cochrane Database Syst Rev. 2007;17:CD006088.

11. Cranswick N, McGillivray G. Over-the-counter medication in children: Friend or foe? Australian Prescriber. 2001;24:149-151.

12. Centers for Disease Control and Prevention. Infant deaths associated with cough and cold medications - two states, 2005. MMWR Morb Mortal Wkly Rep. 2007;56:1-4.

13. Olivieri D, Giacomelli P, Montella R, Illiano A, Gargano G, Pamparano F. Clinical study of a new drug: Cloperastin. Arch Monaldi. 1983;38:209. [Italian].

14. Takagi K, Fulcuda H, Fuije K, Matsui K, Sato M. Studies on antitussives. IV. Various drugs and $\omega$-(diphenilmetoxy) alkylamine compounds. J Pharm Soc Jap. 1961;81:261.

15. Camisasca E, Catenazzo G, Torri A. Cloperastine in chronic bronchopulmonary diseases. Eur Rev Med Pharmacol Sci. 1983;5:375-378.

16. Fabris C. Cloperastine in the treatment of cough. Eur Rev Med Pharmacol Sci. 1983;5:227-230.

17. Oldini C, Vecchi E. Double blind investigation of the antitussive effectiveness of cloperastine. Curr Ther Res. 1987;42:99-105.

18. Margarini G, Scala M, Nocentini L. Cloperastine in the treatment of persistent chronic cough: A controlled clinical study. JAMA. 1998;10:4. Italian version.

19. Thiekotter PB. Cloperastine versus codeine: Controlled clinical study in outpatients with cough of various origin. Pro Med Arztl Ref. 1987; 43:279-282. [German].

20. Ghiringhelli G. Cloperastine (Z15042) in the treatment of cough in patients with exacerbated COPD: Controlled clinical study. Medical Praxis. 1981;2:290-295.

21. Cicchetti M. Cloperastine syrup (Seki) in the treatment of cough in children. Aggiornamento Pediatrico. 1983;34:325-350. Italian.

22. Svitaylo OA. Efficacy and tolerability of cloperastine fendizoate in the treatment of children and adolescents with cough caused by respiratory infections: Effect on night-time cough and the quality of sleep of patients and parents. A randomised, double-blind, placebocontrolled study. International Journal on Drugs and Therapy. 2005;22: $35-43$.

23. Paul IM, Yoder KE, Crowell KR, et al. Effect of dextrometorphan, diphenhydramine, and placebo on nocturnal sleep quality for coughing children and their parents. Pediatrics. 2004;114:e85-e90.

24. Litovitz T, Manoguerra A. Comparison of pediatric poisoning hazards: An analysis of 3.8 million exposure incidents. A report from the American Association of Poison Control Centers. Pediatrics. 1992; 89(6 Pt 1):999-1006.

25. Graudins A, Fern RP. Acute dystonia in a child associated with therapeutic ingestion of a dextromethorphan containing cough and cold syrup. J Toxicol Clin Toxicol. 1996;34(3):351-352.

26. Knowles SR, Weber E. Dextromethorphan anaphylaxis. J Allergy Clin Immunol. 1998;102:316-317. 
27. Cook J, Stith M, Sahn EE. Bullous mastocytosis in an infant associated with the use of a nonprescription cough suppressant. Pediatr Dermatol. 1996;13:410-414.

28. McCarthy JP. Some less familiar drugs of abuse. Med J Aust. 1971;2: 1078-1081.

29. Hapangama A, Kuruppuarachchi KA. Dextromethorphan abuse. Ceylon Med J. 2008;53:109-110.

30. Seidita F, Pamparana F. Nitossil: A new antitussive. A controlled paediatric study. Aggiornamento Pediatrico. 1983;34:363-370. [Italian].

31. Barbato A, Monciotti C, Del Todesco Frisone G. Cloperastine (Nitossil) in the treatment of barking cough in children. Acta Paediatr.1984; 37:349-357.

32. Zanon P, Mazzocchi P, Karitinos P, Savio G, Oberhauser V. Doubleblind, placebo-controlled study of cloperastine, a new antitussive. Rassegna di Fisiopatologia, Clinica e Riabilitazione Cardiorespiratoria. 1985;7:45-49. [Italian].
33. Aliprandi P, Castelli C, Bernorio S, Dell'Abate E, Carrara M. Levocloperastine in the treatment of chronic nonproductive cough: Comparative efficacy versus standard antitussive agents. Drugs Exp Clin Res. 2004;30:133-141.

34. Allegra L, Bossi R. Clinical trials with the new antitussive levodropropizine in adult bronchitic patients. Arzneimittelforschung. 1988;38: 1163-1166.

35. Linazasoro G, Garmendia MT, Lizaso X. Acute dystonia in a young schizophrenic patient associated with ingestion of a cloperastine containing cough syrup. Parkinsonism Relat Disord. 2000;6:57-58.

\section{Publish your work in this journal}

Therapeutics and Clinical Risk Management is an international, peerreviewed journal of clinical therapeutics and risk management, focusing on concise rapid reporting of clinical studies in all therapeutic areas, outcomes, safety, and programs for the effective, safe, and sustained use of medicines. This journal is indexed on PubMed Central, CAS,
EMBase, Scopus and the Elsevier Bibliographic databases. The manuscript management system is completely online and includes a very quick and fair peer-review system, which is all easy to use. Visit http://www.dovepress.com/testimonials.php to read real quotes from published authors.

Submit your manuscript here: http://www.dovepress.com/therapeutics-and-clinical-risk-management-journal 